\title{
DIRECT: RNA contact predictions by integrating structural patterns
}

\author{
Yiren Jian ${ }^{1,2+}$, Xiaonan Wang ${ }^{1+}$, Jaidi Qiu', Huiwen Wang ${ }^{1}$, Zhichao Liư ${ }^{2}$, Yunjie Zhao ${ }^{1 *}$ and Chen Zeng ${ }^{2^{*}}$
}

\begin{abstract}
Background: It is widely believed that tertiary nucleotide-nucleotide interactions are essential in determining RNA structure and function. Currently, direct coupling analysis (DCA) infers nucleotide contacts in a sequence from its homologous sequence alignment across different species. DCA and similar approaches that use sequence information alone typically yield a low accuracy, especially when the available homologous sequences are limited. Therefore, new methods for RNA structural contact inference are desirable because even a single correctly predicted tertiary contact can potentially make the difference between a correct and incorrectly predicted structure. Here we present a new method DIRECT (Direct Information REweighted by Contact Templates) that incorporates a Restricted Boltzmann Machine (RBM) to augment the information on sequence co-variations with structural features in contact inference.

Results: Benchmark tests demonstrate that DIRECT achieves better overall performance than DCA approaches. Compared to mfDCA and pImDCA, DIRECT produces a substantial increase of 41 and 18\%, respectively, in accuracy on average for contact prediction. DIRECT improves predictions for long-range contacts and captures more tertiary structural features.

Conclusions: We developed a hybrid approach that incorporates a Restricted Boltzmann Machine (RBM) to augment the information on sequence co-variations with structural templates in contact inference. Our results demonstrate that DIRECT is able to improve the RNA contact prediction.
\end{abstract}

Keywords: RNA, Restricted Boltzmann machine, Coevolution, Nucleotide-nucleotide interaction

\section{Background}

RNA molecules play critical roles in various biological processes [1-8]. Therefore, a comprehensive determination of RNA structure is critical to understanding structure-function relationships. Unfortunately, it is still challenging to precisely determine structure from direct experimentation [9]. In response, many computational RNA tertiary structure prediction methods have been developed, including homology or fragments-based prediction (ModeRNA, Vfold, RNAComposer, 3dRNA) [10-16] and simulation-based prediction (SimRNA, Rosetta FARFAR, iFoldRNA, NAST) [17-21]. Using these strategies, sequence and secondary structure information can be used to predict RNA tertiary structures.

\footnotetext{
*Correspondence: yjzhaowh@mail.ccnu.edu.cn; chenz@gwu.edu

${ }^{\dagger}$ Yiren Jian and Xiaonan Wang contributed equally to this work.

${ }^{1}$ Institute of Biophysics and Department of Physics, Central China Normal University, Wuhan 430079, China

${ }^{2}$ Department of Physics, The George Washington University, Washington DC 20052, USA
}

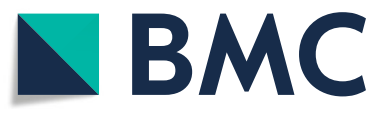

(๑) The Author(s). 2019 Open Access This article is distributed under the terms of the Creative Commons Attribution 4.0 International License (http://creativecommons.org/licenses/by/4.0/), which permits unrestricted use, distribution, and reproduction in any medium, provided you give appropriate credit to the original author(s) and the source, provide a link to the Creative Commons license, and indicate if changes were made. The Creative Commons Public Domain Dedication waiver (http://creativecommons.org/publicdomain/zero/1.0/) applies to the data made available in this article, unless otherwise stated.

The secondary structure is able to define the stem regions and single-stranded loops but leaves RNA tertiary topology unaddressed. Although prediction accuracy has been improved over the years, the tertiary prediction task remains challenging for large RNAs with complex topology. One promising approach is to first predict the tertiary contacts (loop-loop contacts and contacts in junction regions) and then use these interactions to predict the RNA structure. The starting point for this approach is to determine the potential contacts themselves.

One can exploit what is known about nucleotidenucleotide interactions from experimental studies to heuristically provide data about the distances involved in such interactions. One of the most successful methods for contact prediction, based on this approach, is direct coupling analysis (DCA). DCA infers the interacting nucleotides in a sequence from the sequence coevolution across different species [22-33]. A recent mean-field formulation of DCA (mfDCA) provides an efficient computational framework 
to extract direct contact information and has been applied to many RNAs. It has been shown that DCA provides sufficient native intra-domain and inter-domain nucleotidenucleotide contact information for riboswitch and RNAprotein complexes [34-36]. Another inference method called plmDCA, which maximizes the pseudo-likelihood instead of using the mean-field approximation for maximizing the likelihood, improves the contact predictions [37]. In addition to DCA, there are also network-based or machine learning approaches to infer covariation signals from multiple sequence alignments [38-45]. The feature common to these approaches is the exclusive use of evolutionary information extracted from homologous sequences. The prediction accuracy thus depends on accurate multiple sequence alignments of a thousand or more homologous sequences.

An alternative to contact prediction from sequence covariations is to incorporate structural information as well. Skwark et al. applied a pattern-recognition approach to the contact prediction of a residue pair by examining the expected pattern of nearby contacts surrounding the pair [46]. Specifically, a $3 \times 3$ matrix of local contacts is constructed as follows. Each residue of the pair is expanded into a fragment of three residues by including the two neighbors, and all residue-residue contacts between the two fragments form the $3 \times 3$ matrix with element value of 1 for contact and 0 for non-contact. It was found that a contact at the center of the $3 \times 3$ matrix is typically surrounded by three other contacts in the matrix and a noncontact at the center. However, a contact at the center is likely surrounded by no more than one other contact. By incorporating these local contact patterns, this patternrecognition approach is able to improve the prediction of alpha helices and beta strands for protein secondary structures.

However, it is more important and difficult to pinpoint the RNA interactions in loop-loop and junction regions than to identify its secondary structure of base-pair interactions. Existing methods on proteins only consider local structural patterns modeled as statistical potential. This approach ignores global structural features that might be useful in improving the RNA prediction accuracy. Therefore, we introduce a new method that first learns a lookup table of contact weights by a Restricted Boltzmann Machine (RBM) from non-redundant and known RNA structures. Then, this lookup table is used to improve RNA contact prediction obtained from sequence co-evolution by DCA. We call our method Direct Information REweighted by Contact Templates (DIRECT). In a benchmark testing on riboswitch, DIRECT outperforms the state-of-the-art DCA predictions for long-range contacts and loop-loop contacts. Moreover, DIRECT maintains better predictions when the number of available sequences is limited. Here, we examined the accuracy of contact prediction for the 5 RNAs using only 50 randomly chosen homologous sequences that represent about 11 to $43 \%$ of all available sequences for the 5 RNAs.

\section{Results \\ DIRECT achieves better overall performance}

Traditional direct coupling analysis (DCA) for RNA contact prediction has some drawbacks. For one, DCA requires a sufficient number of homologous sequences for accurate sequence co-evolution analysis, which may not be readily available. Moreover, a co-evolving pair of nucleotides can interact within the same molecule (intra-molecule) or across the homodimer interface (inter-molecule) of the RNA. In addition, several unknown factors, other than intra- or inter-molecular interactions, can result in co-evolving pairs and make it difficult to detect the true contacts among the evolving pairs without additional information. One way to overcome this difficulty is to augment the contact detection of a target RNA sequence with additional information on the structural contact template expected of the RNA class to which the target RNA belongs. To this end, we employ a Restricted Boltzmann Machine to learn the contact template of RNAs by using the structures and then improve the contact predictions (Additional file 1: Figure S1).

We used a published riboswitch benchmark dataset to evaluate DIRECT described in Methods (Additional file 1: Table S1, Figure S2) [34]. Six target RNAs are tested as shown in Fig. 1a, b, c, d, e, f. For a given target RNA, the RNA itself and its homologs are removed from the training set. We compare the success rate of mfDCA and DIRECT in predicting the true intra-molecular contacts from the top detected co-evolving pairs (up to top 100). As shown in Fig. 1a, b, c, d, e, f, DIRECT is 5\% 7\% more precise (positive predictive value defined in Methods) than mfDCA for 1Y26, 2GDI, 2GIS, and 3IRW predictions. There is also a slight increase by $2 \%$ for $3 \mathrm{OWI}$ prediction. The improvement continued beyond the top 100 pairs. The only exception is 3VRS, for it differs from others by its higher-order RNA architecture stabilized by pseudoknots with few standard Watson-Crick pairs, which may lead to a low accuracy for contact prediction. The average increase in true positive is $13 \%$. We further evaluated our method DIRECT comparing it to plmDCA, an algorithm that infers the direct coupling using pseudo-likelihood maximization. As shown in Fig. 1g, h, i, j, k, l, DIRECT is $6 \% \sim 8 \%$ more precise (positive predictive value defined in Methods) than plmDCA for 1Y26, 2GIS, and 3OWI predictions. There is also a slight increase by $2 \%$ for 3IRW prediction. Though DIRECT produces lower PPV in 2GDI and 3VRS, DIRECT has $11 \%$ more true positive on average. 


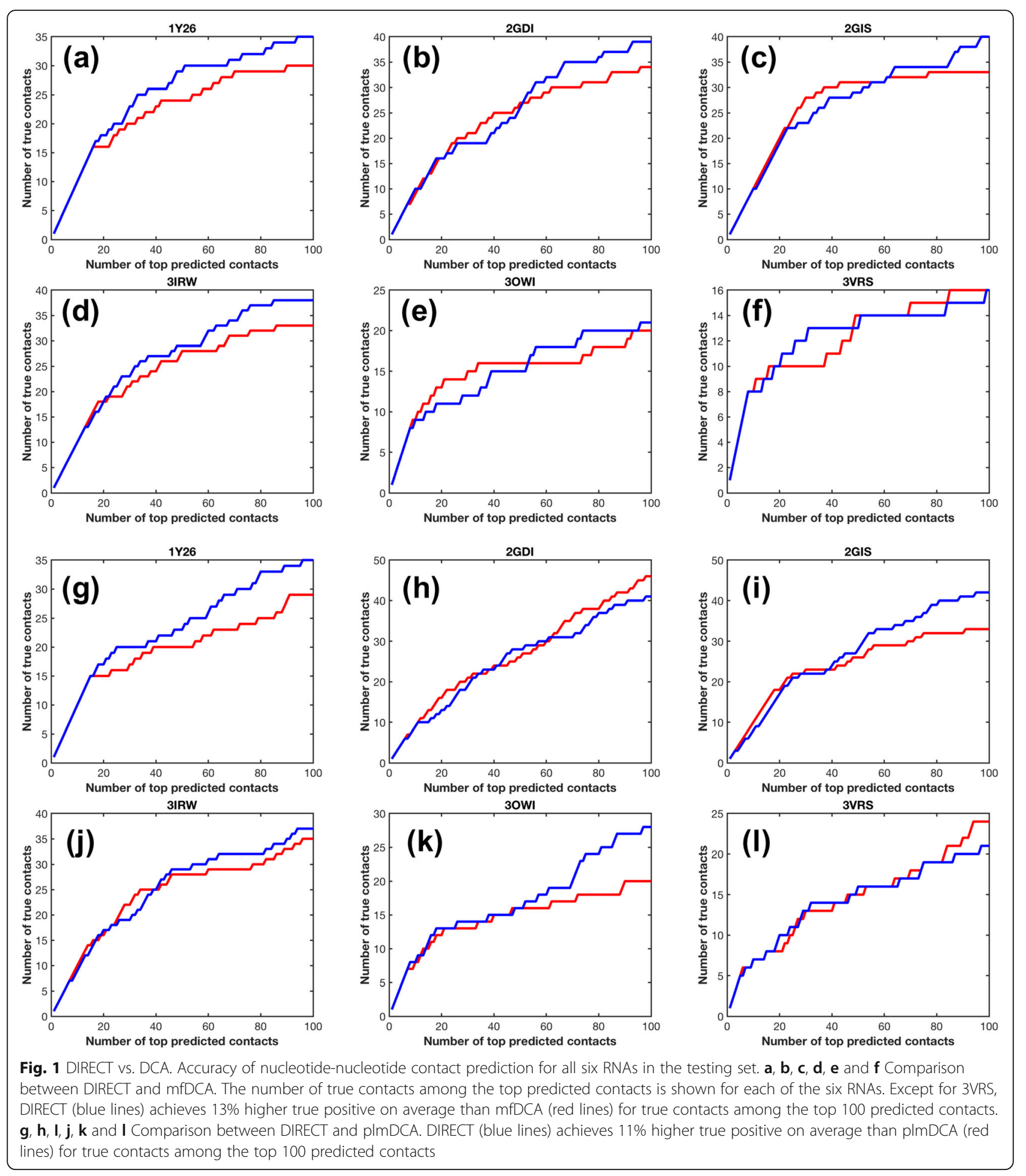

\section{DIRECT improves predictions for long-range contacts}

A contact range measures the sequence distance between the two nucleotides in the contact. Contacts at different ranges convey different information. Short-range contacts in an RNA molecule reflect its local secondary structure. Long-range contacts are base pairs whose contact is based on folding back, loop-loop, or junction interactions. The loop-loop and junction interactions dictate the RNA topology of its structure and are likely to be more useful than secondary structure pairs in structure prediction. A slight improvement in long-range contact prediction, therefore, can have a significant impact on the accuracy 
and speed of RNA tertiary structure modeling because long-range contacts drastically reduce the structural space that needs to be searched for modeling. Prediction based on long-range contacts remains difficult for most traditional methods. DCA predicts more accurately for short- (5 12 nt) and medium-range (13 24 nt) contacts, but less accurately for long-range $(24 \mathrm{nt}+)$ contacts. DIRECT, however, utilizes the structural contact template to re-rank DCA predictions and is able to improve the long-range contact prediction (shown in Additional file 1: Table S2).

\section{DIRECT captures more tertiary structural features}

The interaction types between different RNA secondary structure elements vary significantly. According to Chargaff's second parity rule, base-pair contacts are easier to predict. It remains difficult to predict long-range tertiary contacts. DIRECT is designed to capture the structural contact and improve the prediction accuracy for longrange tertiary contacts. To verify this, we divided the tertiary contacts into four categories: stem-loop, loop-loop, intra stem-stem, and inter stem-stem contacts. The intra stem-stem contacts between two nucleotides in the same stem determine the stem topology such as bending or twisting. On the other hand, contacts of stem-loop, loop-loop, and inter stem-stem can be used as distance constraints on the RNA tertiary fold.

In Additional file 1: Table S3, it can be seen that the largest improvement of predictions by DIRECT lies in tertiary structural contacts. The correct prediction of base pairs can determine RNA secondary structure. The prediction accuracies of base pairs are similar between DCA and DIRECT. These results show that DCA already performs well for base-pair prediction. In contrast, DIRECT improves contacts involving tertiary interactions are improved. There are significant increases of $3 \sim 8$ intra stem-stem contacts correctly predicted for 1Y26, 2GIS, 3OWI, and 3IRW. The intra stem-stem contacts indicate more bending or twisting contacts in these RNA structures. A more pronounced effect can be observed for the other three types of contacts (loop-loop, loop-stem, and inter stem-stem) predictions. In particular, contacts involving loop regions are more accurately predicted. The results show that DIRECT predicts better tertiary fold.

\section{DIRECT identifies more native contacts}

To test if DIRECT is able to identify more native RNA contacts, we ran 4 popular RNA tertiary structure prediction programs (3dRNA, RNAcomposer, simRNA, and Vfold3D) on a given riboswitch to build a number of tertiary structures and evaluated the percentage of top contacts by DIRECT that were actually retained as the structure deviates from the native one. The results of riboswitch 1Y26 are shown in Fig. 2. All other riboswitch tests can be downloaded from our website. We analyzed the Predicted Contacts based on DIRECT prediction (PC), Native Contacts in PC (NC), and RNA Contacts based on predicted structures $(\mathrm{RC})$. Figure 2a shows the correlation between native contacts and RMSDs. The all-atom root-mean-square deviation (RMSD) is measured against the true native structure. The color in Fig. 2a is the percentage of native contacts identified by DIRECT out of top 100 predicted contacts (RC/NC). The predicted structure with the lowest RMSD contains 35 native contacts $(100 \%)$ while the predicted structure with the largest RMSD contains 29 native contacts (83\%). The results show that the native-like structures have much more identified native contacts than the structures with large RMSD values. In addition, we tested the correlation between predicted contacts based on DIRECT prediction and RMSDs if we do not know the native structure. The color in Fig. $2 \mathrm{~b}$ is the percentage of DIRECT predicted contacts out of the top 100 predicted contacts $(\mathrm{RC} / \mathrm{PC})$. The predicted structure with the lowest RMSD contains 40 predicted contacts $(40 \%)$ while the predicted structure with the largest RMSD contains 32 predicted contacts (32\%). The results also show that native-like structures have much more predicted contacts by DIRECT. All results suggest that DIRECT is able to identify more native contacts that define the true RNA tertiary structure.

\section{DIRECT improves RNA contact predictions using non- redundant RNA training sets}

We then used another three non-redundant RNA training sets to evaluate the prediction accuracy of DIRECT. The non-redundant RNA training set 2 contains all the representative high-quality structures of 147 classes with length from $50 \mathrm{nt}$ to $120 \mathrm{nt}$. The homology sequence or structure between training and testing sets were removed to ensure that RNAs in the training and testing sets have no sequence and structural overlap. As shown in Additional file 1: Figure S3, DIRECT is $21 \%$ 95\% and $-4 \% \sim 60 \%$ more precise (positive predictive value) than mfDCA and plmDCA, respectively, for 1Y26, 2GDI, 2GIS, 3IRW, 3OWI, and 3VRS predictions. Taken together, these results suggest that DIRECT is able to improve RNA contact predictions by learning structure template from more known structures as in the RNA non-redundant training set.

Another two non-redundant RNA training sets are as follows: (1) non-redundant RNAs with length from $50 \mathrm{nt}$ to $120 \mathrm{nt}$ without any riboswitch structure (training set 3 in Methods Section), and (2) all non-redundant RNAs without any riboswitch structure (training set 4 in Method Section). The results showed an average accuracy increase of 15 and $4 \%$ compared to mfDCA and 

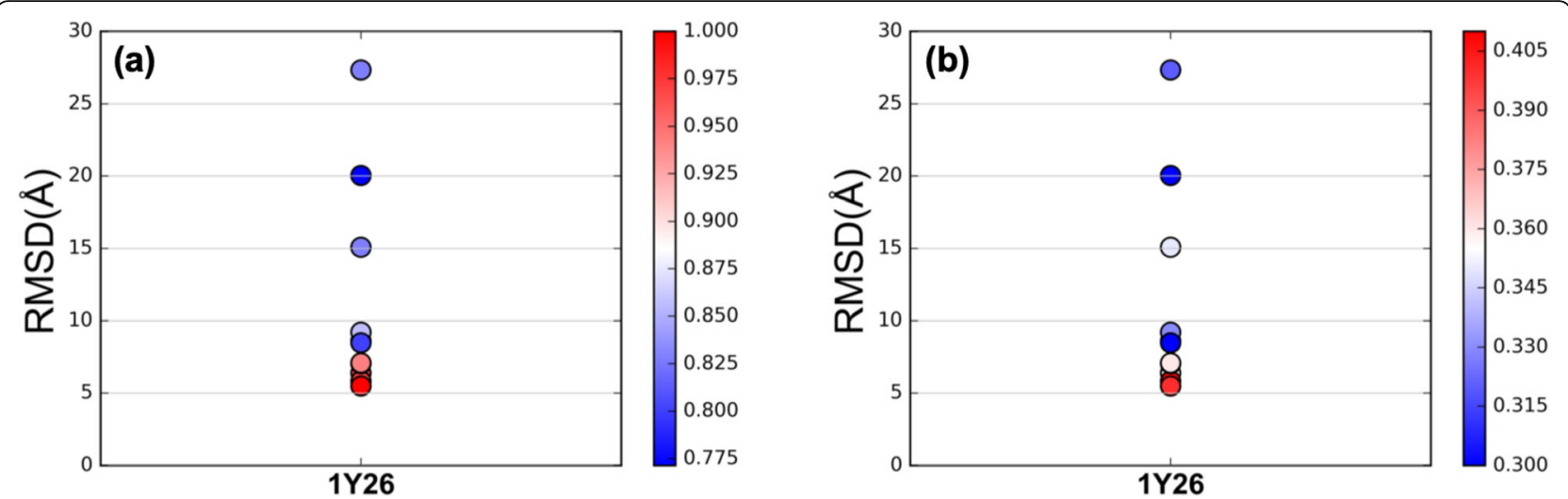

Fig. 2 The contact and RMSD distributions in different RNA structures. The 11 structures are generated by 3dRNA, RNAcomposer, simRNA, and Vfold3D. a shows the correlation between native contacts and RMSDs. The predicted structure with the lowest RMSD contains 35 native contacts (100\%) while the predicted structure with the largest RMSD contains 29 native contacts (83\%). The color in (b) is the percentage of DIRECT predicted contacts out of the top 100 predicted contacts (RC/PC). The predicted structure with the lowest RMSD contains 40 predicted contacts (40\%) while the predicted structure with the largest RMSD contains 32 predicted contacts (32\%). The results suggest that DIRECT is able to identify more native contacts than non-native models with large RMSDs

plmDCA using training set 3 , and 7 and $11 \%$ using the training set 4 . The predictions clearly indicate that there are indeed generic and useful RNA structural features discerned by DIRECT that can improve contact prediction for a specific class of RNA.

\section{DIRECT achieves reliable prediction of conserved contacts} The hypothesis of direct coupling analysis stipulates that co-evolving nucleotides in an RNA molecule may form intra-molecular contacts to support its structure and function. DCA thus aims to disentangle the direct pairwise couplings from indirect correlations of the sequence variations. While highly conserved contacts are critical for RNA structural stability and function, their detection by DCA may be difficult due to insufficient information on variations. To examine if DIRECT can improve the prediction in this case, we divided nucleotides into different types based on their conservation scores calculated by the ConSurf program [47]. The continuous conservation scores are first divided into a discrete scale of 9 grades and the predicted contacts are then classified into three categories: variable contacts (both nucleotides in grade 1-3), conserved contacts (both nucleotides in grade 7-9), and other contacts. As shown in Fig. 3, DIRECT improves the prediction for variable contacts in 1Y26, 2GIS, and 3IRW as well as other contacts in 1Y26, 2GDI, 2GIS, and 3OWI. Although slight improvements observed, it is clear that additional information beyond sequence variation and the structural template is required to achieve a reliable prediction for conserved contacts.

\section{Discussion}

Previous research suggests the number of sequences should be more than three times the length of the molecule for reliable contact prediction [28]. However, many RNA families do not satisfy this condition. While loosening the criterion for homology may result in more sequences, this approach inevitably leads to low accuracy in contact prediction. It remains challenging to extract evolutionary information from an insufficient number of sequences. To check if DIRECT can address the issue of insufficient sequences, we performed contact prediction on 5 target riboswitches using only 50 randomly chosen sequences. The lengths of the 5 RNAs range from 52 to 92 nucleotides and already exceed 50 , the number of sequences used. The results in Additional file 1: Table S4 show that DIRECT outperforms DCA with an average increase of $12 \%$ in prediction precision suggesting that DIRECT can improve predictions even when the number of homologous sequences is insufficient.

To investigate the predictive accuracy on different structural templates, we incorporated a Restricted Boltzmann Machine (RBM) to augment the information on sequence co-variations with four different training sets in contact inference. The contacts learned by Restricted Boltzmann Machine fall mainly into two categories (Additional file 1: Figure S4). One is about the longrange contacts of loop-loop interactions, for example, the loop-loop contacts of A-riboswitch (PDB code: 1Y26), TPP riboswitch (PDB code: 2GDI), SAM-I riboswitch (PDB code: 2GIS), and c-di-GMP riboswitch (PDB code: 3IRW). The other one is about the contacts in junction regions. The contacts of glycine riboswitch (PDB code: $3 \mathrm{OWI}$ ) and fluoride riboswitch (PDB code: 3VRS) define the junction orientations. Unlike local pattern recognition, the global indicator in terms of looploop or junctions contacts is more robust in capturing the folding topology of the entire structure beyond some 

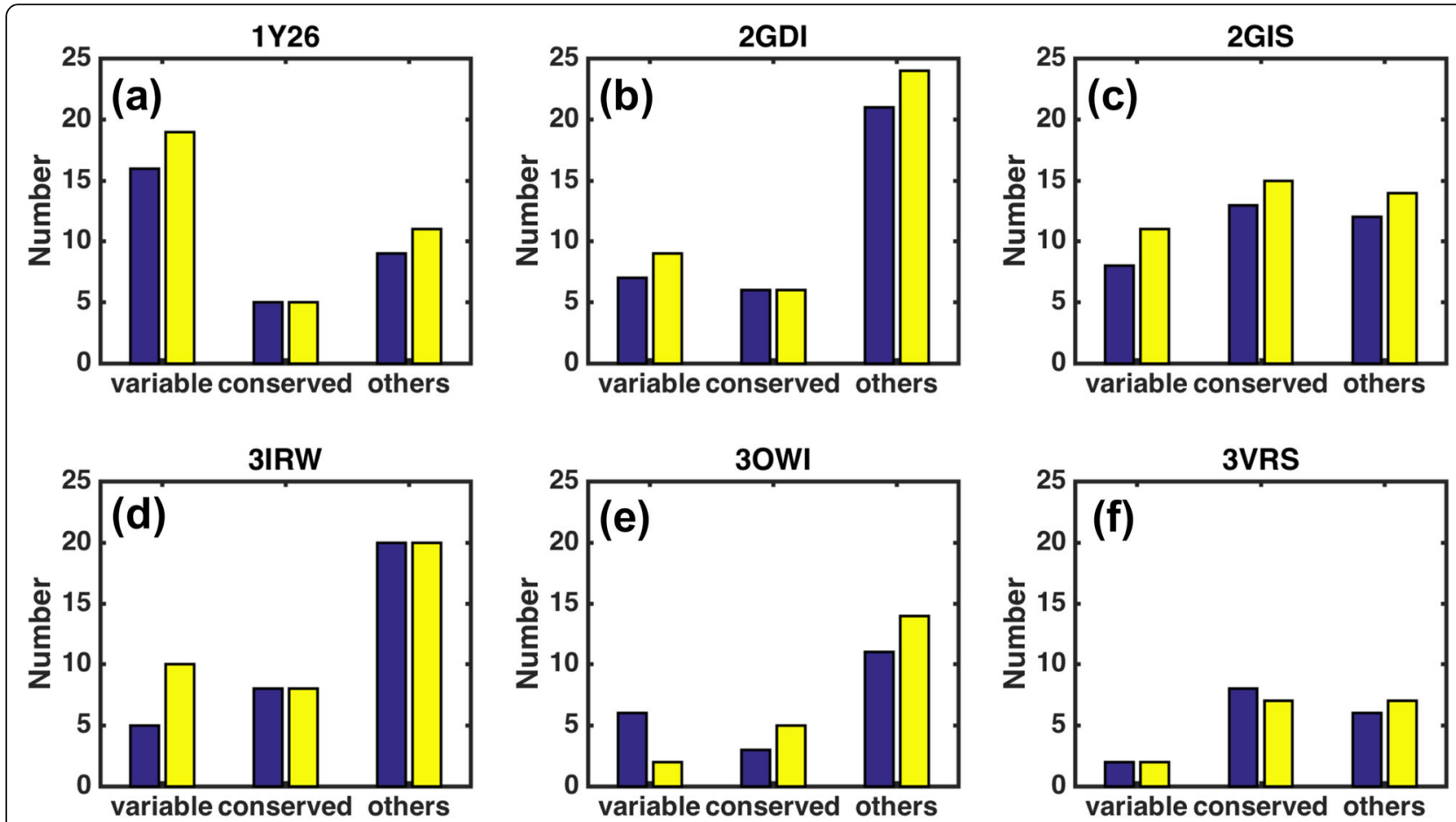

Fig. 3 The number of correctly predicted contacts according to the conservation grades of the two nucleotides involved in the contact for all tested riboswitch RNAs. The contacts are divided into variable type (both nucleotides with conservation-grade 1-3), conserved type (both nucleotides with conservation-grade 7-9), and others, respectively. $\mathbf{a}, \mathbf{b}, \mathbf{c}, \mathbf{d}, \mathbf{e}$, and $\mathbf{f}$ The performance of DIRECT (yellow) in comparison to DCA (blue) is considerably better for the variable contacts and only slightly improved for the conserved contacts

particular parts. DIRECT is able to successfully identify the RNA contact with an average PPV around 0.6 in top 30 predicted contacts (Additional file 1: Figure S8).

\section{Conclusions}

In summary, we developed a hybrid approach that incorporates a Restricted Boltzmann Machine (RBM) to augment the information on sequence co-variations with structural templates in contact inference. Our results demonstrated a 41 and 18\% precision increase for RNA contact prediction in comparison to the mfDCA and plmDCA when structural templates are utilized. In fact, our approach establishes a straightforward framework that can incorporate any additional information, such as NMR spectroscopy data, by training a corresponding Restrictive Boltzmann Machine to further improve the prediction on RNA contacts.

\section{Methods}

\section{Inference workflow}

DIRECT (Direct Information REweighted by Contact Templates) improves the prediction of tertiary contacts by using both sequence and structure information. Figure 4 illustrates the workflow of DIRECT. First, the corresponding RNA multiple sequence alignment (MSA) is extracted from Rfam database. Second, the traditional direct- coupling analysis (DCA) predicts the tertiary contacts from sequence coevolution in MSA. Third, contact weighs are calculated using structural templates trained by Restricted Boltzmann Machine (RBM). Then, DIRECT reweighs the $\mathrm{mfDCA} / \mathrm{plmDCA}$ contact predictions. The inference framework consists of completely hierarchical modules and thus offers the flexibility to incorporate more sequences and structures that may become available in the future, as well as further improved DCA methods for enhanced performance.

\section{Restricted Boltzmann machine (RBM)}

The Restricted Boltzmann Machine (RBM) is a graphical model for unsupervised learning that can extract features from the input data [48]. RBM has a visible layer and a hidden layer. The restriction is that units in the visible layer only interact with units from the hidden layer. This network structure leads to a factorized probability for observing a given configuration, which in turn further simplifies the learning process. The energy of an $\mathrm{RBM}$ is given by

$$
E(v, h \mid W, b, c)=-b^{T} v-c^{T} h-h^{T} W v
$$

where $\mathrm{W}$ is the connection weight matrix between visible 


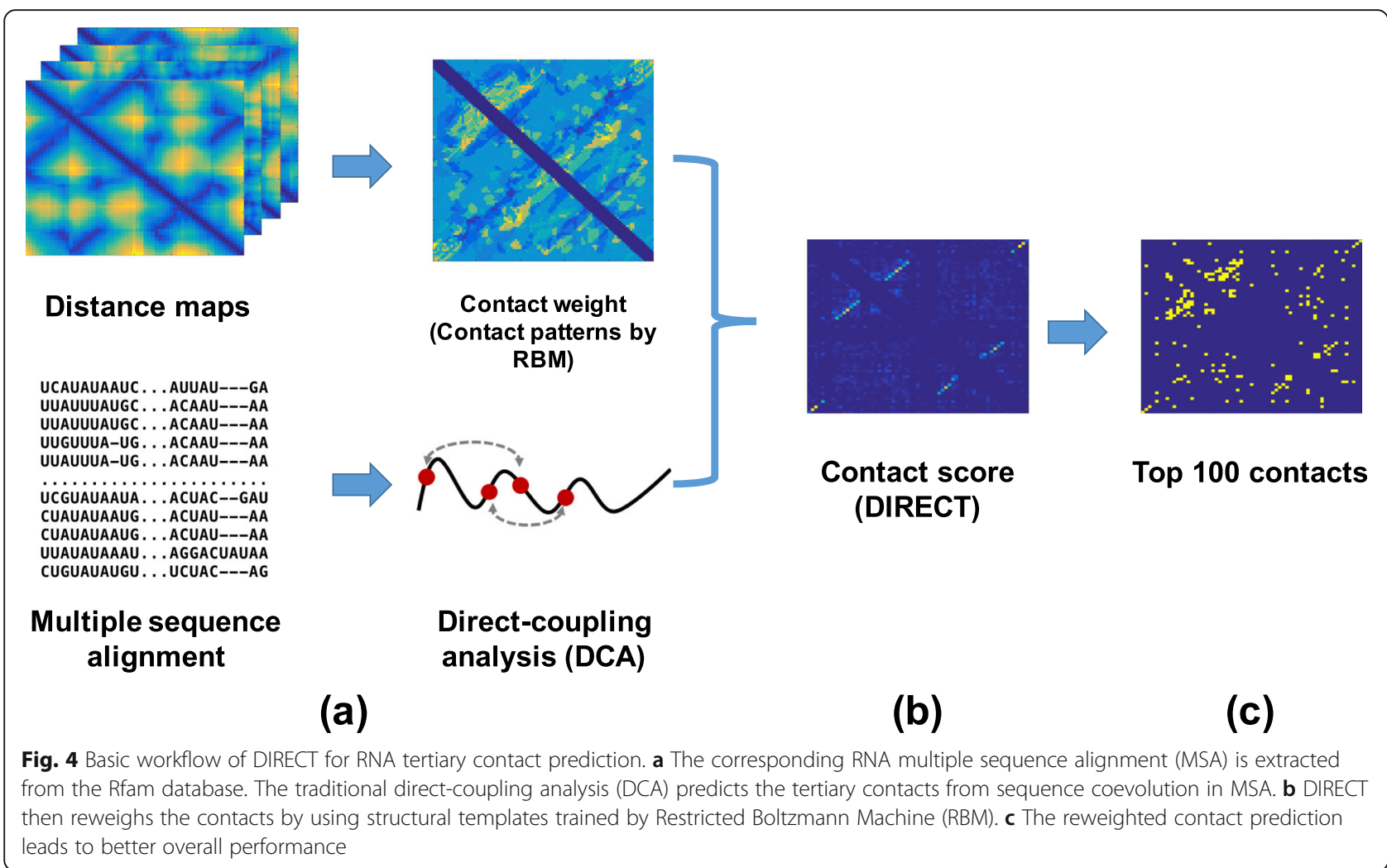

$\mathrm{v}$ and hidden units $\mathrm{h}$. b, c are bias units as offsets. The probability of having a given $\mathrm{v}, \mathrm{h}$ is then

$$
\begin{aligned}
& \mathrm{p}(\mathrm{v}, \mathrm{h} \mid \mathrm{W}, \mathrm{b}, \mathrm{c})=\frac{1}{\mathrm{z}(\mathrm{W}, \mathrm{b}, \mathrm{c})} \mathrm{e}^{-\mathrm{E}(\mathrm{v}, \mathrm{h} \mid \mathrm{W}, \mathrm{b}, \mathrm{c})} \\
& \mathrm{z}(\mathrm{W}, \mathrm{b}, \mathrm{c})=\sum_{\mathrm{v}, \mathrm{h}} \mathrm{e}^{-\mathrm{E}(\mathrm{v}, \mathrm{h} \mid \mathrm{W}, \mathrm{b}, \mathrm{c})}
\end{aligned}
$$

where $z(W, b, c)$ is the partition function that sums up all possible $\mathrm{v}$ and $\mathrm{h}$. The RBM is trained through stochastic gradient descent (SGD) on negative loglikelihood of the empirical data. $\mathrm{L}(\mathrm{W}, \mathrm{c}, \mathrm{b}, \mathrm{T})$ is defined as the loss function, which we want to minimize during SGD:

$$
\mathrm{L}(\mathrm{W}, \mathrm{c}, \mathrm{b}, \mathrm{T})=-\frac{1}{\mathrm{~N}} \sum_{\mathrm{v} \in \mathrm{T}} \log \mathrm{P}(\mathrm{v} \mid \mathrm{W}, \mathrm{b}, \mathrm{c})
$$

where $\mathrm{P}(\mathrm{v} \mid \mathrm{W}, \mathrm{b}, \mathrm{c})$ is given by

$$
\mathrm{P}(\mathrm{v} \mid \mathrm{W}, \mathrm{b}, \mathrm{c})=\sum_{\mathrm{h}} \mathrm{p}(\mathrm{v}, \mathrm{h} \mid \mathrm{W}, \mathrm{b}, \mathrm{c})
$$

$\mathrm{T}$ above is a set of samples from the empirical data. By minimizing the loss function, we can update the parameters $\mathrm{W}, \mathrm{b}, \mathrm{c}$ according to the equations below:

$$
\begin{aligned}
& \mathrm{W}=\mathrm{W}-\frac{\partial \mathrm{L}(\mathrm{W}, \mathrm{b}, \mathrm{c}, \mathrm{T})}{\partial \mathrm{W}} \\
& \mathrm{b}=\mathrm{b}-\frac{\partial \mathrm{L}(\mathrm{W}, \mathrm{b}, \mathrm{c}, \mathrm{T})}{\partial \mathrm{b}}
\end{aligned}
$$

$$
\mathrm{c}=\mathrm{c}-\frac{\partial \mathrm{L}(\mathrm{W}, \mathrm{b}, \mathrm{c}, \mathrm{T})}{\partial \mathrm{c}}
$$

\section{Contact definition and evaluation criteria}

Two nucleotides are considered in contact if they contain a pair of heavy atoms, one from each nucleotide, less than a pre-defined cutoff [49-51]. Previous work indicated that $8 \AA$ can serve as a reliable contact cutoff for RNA tertiary structural study [34, 35]. To compare DIRECT with earlier methods, we use the same reliable contact distance cutoff of $8 \AA$ as in previous studies [34, 35]. A-form RNA rises $2.6 \AA$ per base pair; the stacking interaction is thus small if the distance of two nucleotides is larger than $8 \AA$. Since adjacent nucleotides in a sequence are always in contact, we only consider contacts between nucleotides that are separated by more than four nucleotides in a sequence to measure tertiary contacts of interest. To evaluate the quality of a prediction, we compute the positive predictive value (PPV) as follows.

$$
P P V=\frac{|T P|}{|T P|+|F P|}
$$

where TP (FP) denotes the true (false) positive and stands for the number of true (false) positives. 


\section{Training and testing sets}

Riboswitch is a regulatory portion of a messenger RNA. When binding with a small ligand, this regulatory segment will regulate the translation of the entire mRNA. In this study, we constructed four different training sets ranging from containing no homologous riboswitch with similar RNA size to strictly no riboswitch with all RNA sizes. This is to ensure that there is no data leakage between the training set and testing set so that the features captured by DIRECT are generic and useful RNA template patterns. Details of the datasets are as follows.

(1) RNA non-redundant training set 1 . To generate a representative set of riboswitch families for our study, we systematically selected riboswitch families from the Rfam database. The ten representative riboswitches in the training set are shown in Additional file 1: Table S5. We analyzed the sequence identity value (calculated by CLUSTALW, http://www.genome.jp/tools-bin/clustalw) and structural similarity via RMSD value (calculated by PyMOL, www.pymol.org) between each pair of RNAs in the training set (Additional file 1: Table S6). The large values for sequence diversity and RMSD suggest that the RNAs in the training set share little similarity in sequence and structure.

(2) RNA non-redundant training set 2 . We collected all the representative high-quality structures with resolution $\leq 3.0 \AA$ of 147 classes of RNA 3D Hub nonredundant RNA set (version 3.21) with length from 50 nt to 120 nt [52]. RNA 3D Hub ensured that sequence identity between any two sequences is $<95 \%$. It is noted that existing methods for RNA tertiary structure prediction (for example, RASP and 3dRNA) also used a sequence identity of $95 \%$ to reduce redundancy in training set $[14,15,53]$. RNAs that share sequence or structure homology in training and testing sets were removed from the training set. These steps ensure that structures in the training set and testing sets have a similar size but no sequence and structural overlap.

(3) RNA non-redundant training set 3 . We collected all the representative high-quality structures in RNA non-redundant training set 2 , then removed all riboswitch structures in this training set. These steps ensure no riboswitch structure in the training set.

(4) RNA non-redundant training set 4. Unlike RNA non-redundant training set 3 that collected similar size RNAs as a testing set (from $50 \mathrm{nt}$ to $120 \mathrm{nt}$ ), we collected all the representative high-quality tertiary structures with resolution $\leq 3.0 \AA$ of 1023 classes of RNA 3D Hub non-redundant RNA set (version 3.21). Then, we also removed all the riboswitch structures in this training set. These steps ensure no riboswitch structure in the training set. The lists of RNA nonredundant training set 3 and 4 can be downloaded from our website.
For the testing set, we used the published testing dataset including six riboswitches (Additional file 1: Table S1) [34].

\section{Weight of structural information learned by RBM for prediction of riboswitch}

The Restricted Boltzmann Machine (RBM) is used to extract the contact knowledge from riboswitch structures in the training set (Fig. 5).

Step 1: Prepare the training set (Fig. 5a and b). Riboswitch structures in the training set are converted into contact maps by applying the distance cutoff of $8 \AA$. The lengths of the testing riboswitches range from 52 to 94 nucleotides. For the convenience of integrating the templates of structural information, all distance maps are resized by linear interpolation into the same size of $100 \times 100$ pixels before applying the distance cutoff of 8 $\AA$. Image resizing is widely used in deep learning communities to train a machine with fixed input of picture sizes. One of the popular architecture of convolutional neural networks, VGG-16, is trained with over 14 million images belonging to 1000 classes [54]. All images are resized into a size of $224 \times 244 \times 3$ (RGB image) before being fed into the machine. VGG-16 achieves $70.5 \%$ accuracy for top 1 prediction and 90\% accuracy for top 5 predictions. Following a similar consideration and given that the riboswitches in our training set have varying lengths of 54 to 94 nucleotides, we also resized distance maps into a fixed size of $100 \times 100$ and then converted it into a contact map using a cutoff of $8 \AA$. The resizing done by the linear interpolation will keep the spatial information invariant between nucleotides. For example, the distance between nucleotide 1 and 4 in an RNA with 50 nucleotides will stay the same between position 1 and 8 after we resize the distance matrix into $100 \times 100$. The contact patterns are almost identical between original and resized ones (Additional file 1: Figure S5) since one is a coarse-grained version of the other. These results show that the global features and local patterns are kept during the resizing. To remove the overlap between the training set and testing set, we exclude all homologous training structures with respect to the riboswitch structure in the testing set for each prediction. To be more precise for this blind test, when predicting each of the six riboswitches in the test set, the targeting riboswitch and all its homologs are removed from the training set. RBM learned six different weights of structural information for the six riboswitches. We converted the lower triangle contact maps into a onedimensional array with one channel per contact (as 1) or non-contact (as 0). The elements of this onedimensional array will be fed into the visible units of RBM. Thus, in our six different tests, the number of visible units of RBM is determined by the size of the contact map (or in other words, the number of nucleotides in 


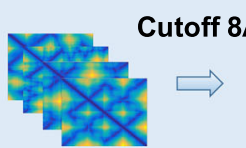

Distances

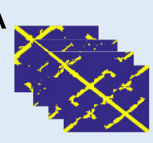

Contacts

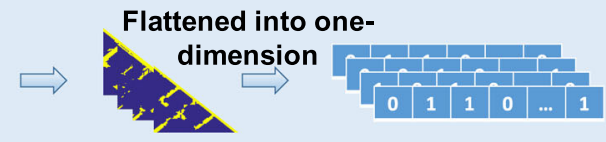

Lower-triangle

(a)

\section{Prepare contact training set}

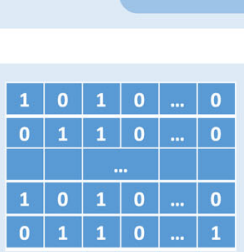

Contact information from different RNAs

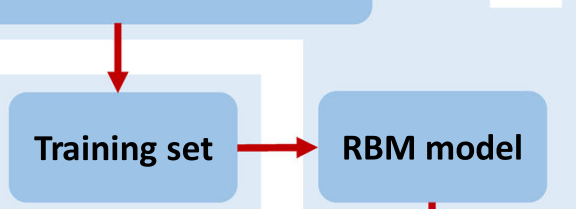

(b)

(c)

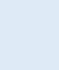

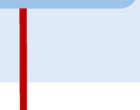

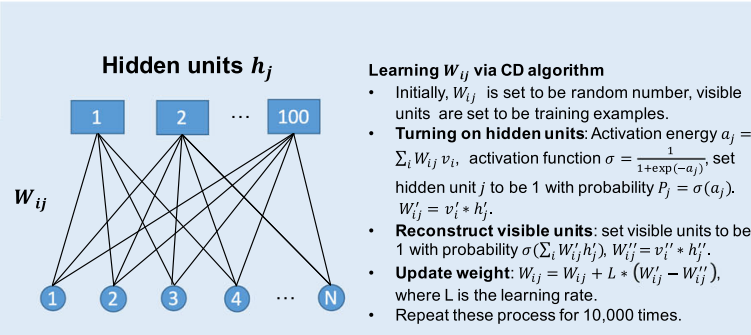

Visible units $v_{i}$

(e)

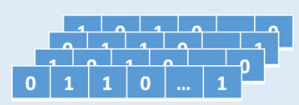

\& normalization
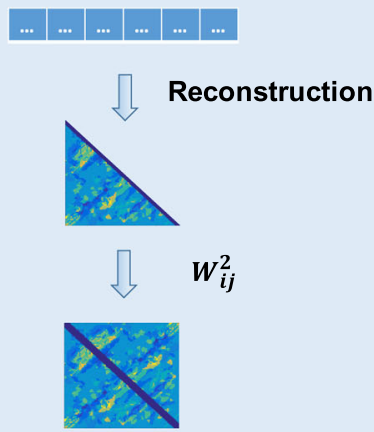

Summation

Contact weight

$W_{i j}^{2}$
Wis units are set to be training examples.
Turning on hidden units: Activation energy $a_{j}=$ $\sum_{i} W_{i j} v_{i}$, activation function $\sigma=\frac{1}{1+\exp \left(-a_{j}\right)}$, set hidden unit $j$ to be 1 with probability $P_{j}=\sigma\left(a_{j}\right)$. $W_{i j}^{\prime}=v_{i}^{\prime} * h_{j}^{\prime}$. Update weight: $W_{i j}=W_{i j}+L *\left(W_{i j}^{\prime}-W_{i j}^{\prime \prime}\right)$, Repeat these process for 10,000 times. 
RBM's belief for the most common structure of riboswitches. What the RBM learned in the previous step is the hidden connections between hidden representations and contact patterns (visible representations). Gibbs sampling method is the widely used approach to get samples from an energy-based model. We turned the model into a generative mode to produce visible contact patterns through a Gibbs sampling process. To get the unbiased samples from the model we learned in the previous step, we need to run Gibbs sampling for a long time for convergence. 10, 000 samples generated by the model at this stage are converged.

Step 4: Contact weight learned by RBM (Fig. 5e). We counted the contact frequency for each nucleotide among these 5000 structures and took this frequency as the final weight matrix learned by RBM on the structure information of the riboswitch.

\section{Direct coupling analysis}

The direct coupling analysis (DCA) is performed to infer the interacting nucleotides from sequence coevolution across different species [22, 35, 56]. We first removed the sequences with gaps of more than $50 \%$ in multiple sequence alignment (MSA) and then calculated the amino acid frequencies for singlenucleotide and a pair of nucleotides. The direct couplings that indicate the interaction strength between two sites are defined as

$$
D I_{i j}=\sum_{A B} P_{i j}^{d}(A, B) \ln \frac{P_{i j}^{d}(A, B)}{f_{i}(A) f_{j}(B)}
$$

with the help of an isolated two-site model

$$
P_{i j}^{d}(A, B)=\exp \left\{e_{i j}(A, B)+\tilde{h}_{i}(A)+\tilde{h}_{j}(B)\right\} / Z_{i j}
$$

$\tilde{h}_{i}(A)$ and $\tilde{h}_{j}(B)$ are defined by the empirical singlenucleotide frequency $f_{i}(A)=\sum_{B} P_{i j}^{d}(A, B)$ and $f_{j}(B)$ $=\sum_{A} P_{i j}^{d}(A, B)$. Mean-field DCA (mfDCA) is done by a simple mean-field approximation, see Morcos et al. (Morcos, et al., 2011) for details. Ekeberg also proposes a method called plmDCA using pseudo-likelihood maximization for inferring direct coupling $[37,57]$. We used the downloaded versions of mfDCA and plmDCA algorithms. The mfDCA was downloaded from http://dca. rice.edu/portal/dca/download. The plmDCA was downloaded from the Marks lab at Harvard Medical School (http://evfold.org/evfold-web/code.do).

\section{Direct information scores reweighted by structural contact frequency}

The final contact prediction is DI scores reweighted by structural information learned by RBM with better contact prediction accuracy.

$$
D I R E C T=D I \times W^{2}
$$

where DI is the direct information by direct coupling analysis, $\mathrm{W}$ is RBM-based structural contact frequency. Among the different powers of $\mathrm{W}$ considered (up to the 4th power), we finally selected the 2 nd power of $\mathrm{W}$ as in Eq. (12) to balance the contributions from both patterns of sequence evolution and RBM-based structural contact frequency.

\section{Tertiary structure prediction}

We predicted RNA tertiary structures using 3dRNA, RNAcomposer, simRNA and Vfold3D [11, 14, 15, 17, 58]. For each RNA structure prediction, we used the corresponding sequence and secondary structure on the RNA structure modeling servers. All tertiary structures are predicted automatically.

\section{Regularization}

Regularization is a strategy that aims to reduce the generalization errors [59]. Most machine learning methods add restrictions on the parameters. For example, L1 and L2 regularization are adding a cost function that penalizes high-value weights to prevent overfitting. The weight of RBM is a matrix of $4951 \times 101$ (connecting the 4950 visible units and 100 hidden units, the one extra unit on each side is the bias unit). Although we did not implement the regularization in our model training, the obtained weights shown in Additional file 1: Figure S7 did not take extreme values associated with overfitting.

\section{Supplementary information}

Supplementary information accompanies this paper at https://doi.org/10. 1186/s12859-019-3099-4.

Additional file 1. Supplementary material, including all supplementary figures and supplementary tables.

\section{Abbreviations}

CD: Contrastive-Divergence; DCA: Direct coupling analysis; DI: Direct information; DIRECT: Direct Information REweighted by Contact Templates; FP: False positive; inter-molecule: Across the homodimer interface; intramolecule: Interact within the same molecule; mfDCA: Mean-field direct coupling analysis; MSA: Multiple sequence alignment; NC: Native Contacts; NMR: Nuclear Magnetic Resonance; PC: Predicted Contacts; plmDCA: Pseudolikelihood maximizes direct coupling analysis; PPV: Positive predictive value; RBM: Restricted Boltzmann Machine; RC: RNA Contacts; RMSD: Root-meansquare deviation; SGD: Stochastic gradient descent; TP: True positive

Acknowledgments

Not applicable. 


\section{Authors' contributions}

YJ performed most computational analysis under the supervision of $Y Z$ and $C Z$. XW, JQ, HW and ZL performed the non-redundant training and computational analysis under the supervision of $\mathrm{YZ}$. $\mathrm{YZ}$ and $\mathrm{CZ}$ supervised the overall study and wrote the paper. All authors read and approved the manuscript.

\section{Funding}

This work is supported by the National Natural Science Foundation of China 11704140, Natural Science Foundation of Hubei 2017CFB116, and selfdetermined research funds of CCNU from the colleges' basic research and operation of MOE CCNU19QD008 to YZ. The funders had no role in the design of the study, collection, analysis, interpretation of data, and writing the manuscript.

\section{Availability of data and materials}

The codes and dataset are available at https://zhaolab.com.cn/DIRECT/.

\section{Ethics approval and consent to participate}

Not applicable.

\section{Consent for publication}

Not applicable.

\section{Competing interests}

The authors declare that they have no competing interests.

Received: 8 March 2019 Accepted: 13 September 2019

\section{Published online: 15 October 2019}

\section{References}

1. Sharma U, Conine CC, Shea JM, Boskovic A, Derr AG, Bing XY, et al. Biogenesis and function of tRNA fragments during sperm maturation and fertilization in mammals. Science. 2016:351:391-6.

2. Goodarzi H, Liu X, Nguyen HC, Zhang S, Fish L, Tavazoie SF. Endogenous tRNA-derived fragments suppress breast Cancer progression via YBX1 displacement. Cell. 2015;161:790-802.

3. Esteller M. Non-coding RNAs in human disease. Nat Rev Genet. 2011;12: 861-74

4. Lunse CE, Schuller A, Mayer G. The promise of riboswitches as potential antibacterial drug targets. Int J Med Microbiol. 2014;304:79-92.

5. Breaker RR. Riboswitches and the RNA world. Cold Spring Harb Perspect Biol. 2012:4:a003566.

6. Montange RK, Batey RT. Riboswitches: emerging themes in RNA structure and function. Annu Rev Biophys. 2008;37:117-33.

7. Shi M, Lin XD, Tian JH, Chen LJ, Chen X, Li CX, et al. Redefining the invertebrate RNA virosphere. Nature. 2016;540:539-43.

8. Fire A, Xu S, Montgomery MK, Kostas SA, Driver SE, Mello CC. Potent and specific genetic interference by double-stranded RNA in Caenorhabditis elegans. Nature. 1998;391:806-11.

9. Mortimer SA, Kidwell MA, Doudna JA. Insights into RNA structure and function from genome-wide studies. Nat Rev Genet. 2014;15:469-79.

10. Rother M, Milanowska K, Puton T, Jeleniewicz J, Rother K, Bujnicki JM. ModeRNA server: an online tool for modeling RNA 3D structures. Bioinformatics. 2011;27:2441-2.

11. Xu X, Zhao P, Chen SJ. Vfold: a web server for RNA structure and folding thermodynamics prediction. PLoS One. 2014;9:e107504.

12. Popenda M, Szachniuk M, Antczak M, Purzycka KJ, Lukasiak P, Bartol N, et al, Automated 3D structure composition for large RNAs. Nucleic Acids Res. 2012;40:e112.

13. Zhao Y, Gong Z, Xiao Y. Improvements of the hierarchical approach for predicting RNA tertiary structure. J Biomol Struct Dyn. 2011:28:815-26.

14. Zhao Y, Huang Y, Gong Z, Wang Y, Man J, Xiao Y. Automated and fast building of three-dimensional RNA structures. Sci Rep. 2012;2:734.

15. Wang J, Zhao Y, Zhu C, Xiao Y. 3dRNAscore: a distance and torsion angle dependent evaluation function of 3D RNA structures. Nucleic Acids Res. 2015;43:e63.

16. Zhao Y, Wang J, Zeng C, Xiao Y. Evaluation of RNA secondary structure prediction for both base-pairing and topology. Biophysics Reports. 2018;4: $123-32$
17. Boniecki MJ, Lach G, Dawson WK, Tomala K, Lukasz P, Soltysinski T, et al. SimRNA: a coarse-grained method for RNA folding simulations and 3D structure prediction. Nucleic Acids Res. 2016;44:e63.

18. Das R, Karanicolas J, Baker D. Atomic accuracy in predicting and designing noncanonical RNA structure. Nat Methods. 2010;7:291-4

19. Krokhotin A, Houlihan K, Dokholyan NV. iFoldRNA v2: folding RNA with constraints. Bioinformatics. 2015;31:2891-3.

20. Jonikas MA, Radmer RJ, Laederach A, Das R, Pearlman S, Herschlag D, et al. Coarse-grained modeling of large RNA molecules with knowledge-based potentials and structural filters. RNA. 2009;15:189-99.

21. Shi $Y Z$, Jin L, Wang FH, Zhu XL, Tan ZJ. Predicting 3D structure, flexibility, and stability of RNA hairpins in monovalent and divalent ion solutions. Biophys J. 2015;109:2654-65.

22. Morcos F, Pagnani A, Lunt B, Bertolino A, Marks DS, Sander C, et al. Directcoupling analysis of residue coevolution captures native contacts across many protein families. Proc Natl Acad Sci U S A. 2011;108:E1293-301.

23. de Juan D, Pazos F, Valencia A. Emerging methods in protein co-evolution. Nat Rev Genet. 2013;14:249-61.

24. Marks DS, Hopf TA, Sander C. Protein structure prediction from sequence variation. Nat Biotechnol. 2012;30:1072-80.

25. Weigt M, White RA, Szurmant $H$, Hoch JA, Hwa T. Identification of direct residue contacts in protein-protein interaction by message passing. Proc Natl Acad Sci U S A. 2009;106:67-72.

26. Xing S, Li F, Zeng Z, Zhao Y, Yu S, Shan Q, et al. Tcf1 and Lef1 transcription factors establish CD8(+) T cell identity through intrinsic HDAC activity. Nat Immunol. 2016;17:695-703

27. Hopf TA, Colwell LJ, Sheridan R, Rost B, Sander C, Marks DS. Threedimensional structures of membrane proteins from genomic sequencing. Cell. 2012;149:1607-21.

28. Hopf TA, Scharfe CP, Rodrigues JP, Green AG, Kohlbacher O, Sander C, et al. Sequence co-evolution gives $3 \mathrm{D}$ contacts and structures of protein complexes. Elife. 2014;3:e03430.

29. Stein RR, Marks DS, Sander C. Inferring pairwise interactions from biological data using maximum-entropy probability models. PLoS Comput Biol. 2015; 11:e1004182.

30. Marks DS, Colwell LJ, Sheridan R, Hopf TA, Pagnani A, Zecchina R, et al. Protein 3D structure computed from evolutionary sequence variation. PLoS One. 2011;6:e28766.

31. Hopf TA, Morinaga S, Ihara S, Touhara K, Marks DS, Benton R. Amino acid coevolution reveals three-dimensional structure and functional domains of insect odorant receptors. Nat Commun. 2015;6:6077.

32. Ovchinnikov S, Kamisetty H, Baker D. Robust and accurate prediction of residue-residue interactions across protein interfaces using evolutionary information. Elife. 2014;3:e02030.

33. Ovchinnikov S, Park H, Varghese N, Huang PS, Pavlopoulos GA, Kim DE, et al. Protein structure determination using metagenome sequence data. Science. 2017:355:294-8.

34. De Leonardis E, Lutz B, Ratz S, Cocco S, Monasson R, Schug A, et al. Directcoupling analysis of nucleotide coevolution facilitates RNA secondary and tertiary structure prediction. Nucleic Acids Res. 2015;43:10444-55.

35. Weinreb C, Riesselman AJ, Ingraham JB, Gross T, Sander C, Marks DS. 3D RNA and functional interactions from evolutionary couplings. Cell. 2016;165: 963-75.

36. Wang J, Mao K, Zhao Y, Zeng C, Xiang J, Zhang Y, et al. Optimization of RNA 3D structure prediction using evolutionary restraints of nucleotidenucleotide interactions from direct coupling analysis. Nucleic Acids Res. 2017:45:6299-309.

37. Ekeberg M, Hartonen T, Aurell E. Fast pseudolikelihood maximization for direct-coupling analysis of protein structure from many homologous aminoacid sequences. J Comput Phys. 2014;276:341-56.

38. Wang S, Sun S, Li Z, Zhang R, Xu J. Accurate De novo prediction of protein contact map by ultra-deep learning model. PLoS Comput Biol. 2017;13: e1005324.

39. Di Lena $P$, Nagata K, Baldi P. Deep architectures for protein contact map prediction. Bioinformatics. 2012;28:2449-57.

40. Ma J, Wang S, Wang Z, Xu J. Protein contact prediction by integrating joint evolutionary coupling analysis and supervised learning. Bioinformatics. 2015; 31:3506-13.

41. Jones DT, Singh T, Kosciolek T, Tetchner S. MetaPSICOV: combining coevolution methods for accurate prediction of contacts and long range hydrogen bonding in proteins. Bioinformatics. 2015;31:999-1006. 
42. Eickholt J, Cheng J. Predicting protein residue-residue contacts using deep networks and boosting. Bioinformatics. 2012;28:3066-72.

43. Li Y, Huang C, Ding L, Li Z, Pan Y, Gao X. Deep learning in bioinformatics: introduction, application, and perspective in the big data era. Methods. 2019;166:4-21

44. Tang B, Pan Z, Yin K, Khateeb A. Recent advances of deep learning in bioinformatics and computational biology. Front Genet. 2019;10:214.

45. Lan K, Wang DT, Fong S, Liu LS, Wong KKL, Dey N. A survey of data mining and deep learning in bioinformatics. J Med Syst. 2018;42:139.

46. Skwark MJ, Raimondi D, Michel M, Elofsson A. Improved contact predictions using the recognition of protein like contact patterns. PLoS Comput Biol. 2014;10:e1003889.

47. Ashkenazy H, Abadi S, Martz E, Chay O, Mayrose I, Pupko T, et al. ConSurf 2016: an improved methodology to estimate and visualize evolutionary conservation in macromolecules. Nucleic Acids Res. 2016:44:W344-50.

48. Hinton GE. A practical guide to training restricted Boltzmann machines. Momentum. 2012;9:599-619.

49. Zhao Y, Jian Y, Liu Z, Liu H, Liu Q, Chen C, et al. Network analysis reveals the recognition mechanism for dimer formation of bulb-type lectins. Sci Rep. 2017;7:2876.

50. Chen H, Zhao Y, Li H, Zhang D, Huang Y, Shen Q, et al. Break CDK2/cyclin E1 interface allosterically with small peptides. PLoS One. 2014;9:e109154.

51. Wang $K$, Jian $Y$, Wang $H$, Zeng C, Zhao Y. RBind: computational network method to predict RNA binding sites. Bioinformatics. 2018;34:3131-6.

52. Leontis NB, Zirbel CL. Nonredundant $3 D$ Structure Datasets for RNA Knowledge Extraction and Benchmarking. In: Leontis N, Westhof E, editors. RNA 3D Structure Analysis and Prediction, vol. 27. Berlin Heidelberg: Springer; 2012. p. 281-98.

53. Capriotti E, Norambuena T, Marti-Renom MA, Melo F. All-atom knowledgebased potential for RNA structure prediction and assessment. Bioinformatics. 2011;27:1086-93.

54. Simonyan K, Zisserman A. Very Deep Convolutional Networks for LargeScale Image Recognition. arXiv:1409.1556. https://arxiv.org/abs/1409.1556.

55. Hinton GE. A Practical Guide to Training Restricted Boltzmann Machines. In: Montavon G, Orr GB, Müller K-R, editors. Neural Networks: Tricks of the Trade, vol. 9. 2nd ed. Berlin, Heidelberg: Springer Berlin Heidelberg; 2012. p. 599-619.

56. Morcos F, Hwa T, Onuchic JN, Weigt M. Direct coupling analysis for protein contact prediction. Methods Mol Biol. 2014;1137:55-70.

57. Ekeberg M, Lovkvist $\mathrm{C}$, Lan $\mathrm{Y}$, Weigt $\mathrm{M}$, Aurell E. Improved contact prediction in proteins: using pseudolikelihoods to infer Potts models. Phy Rev E Stat Nonlin Soft Matter Phys. 2013;87:012707.

58. Biesiada M, Purzycka KJ, Szachniuk M, Blazewicz J, Adamiak RW. Automated RNA 3D structure prediction with RNAComposer. Methods Mol Biol. 2016; 1490:199-215.

59. Wang L, Gordon MD, Zhu J. Regularized Least Absolute Deviations Regression and an Efficient Algorithm for Parameter Tuning. In: Sixth International Conference on Data Mining (ICDM'06): 18-22; 2006. p. 690-700.

\section{Publisher's Note}

Springer Nature remains neutral with regard to jurisdictional claims in published maps and institutional affiliations.

Ready to submit your research? Choose BMC and benefit from:

- fast, convenient online submission

- thorough peer review by experienced researchers in your field

- rapid publication on acceptance

- support for research data, including large and complex data types

- gold Open Access which fosters wider collaboration and increased citations

- maximum visibility for your research: over $100 \mathrm{M}$ website views per year

At $\mathrm{BMC}$, research is always in progress.

Learn more biomedcentral.com/submissions 